\title{
PURELY INFINITE TOTALLY DISCONNECTED TOPOLOGICAL GRAPH ALGEBRAS
}

\author{
HUI LI
}

\begin{abstract}
We give a sufficient condition on totally disconnected topological graphs such that their associated topological graph algebras are purely infinite.
\end{abstract}

\section{INTRODUCTION}

The Elliott program developed rapidly over the last twenty years with the goal of classifying all simple separable nuclear $C^{*}$-algebras by the so-called Elliott's invariants (Notice that different types of classification might have different invariants). In the purely infinite case, Kirchberg in 9] and Phillips in [14 separately showed that all simple separable nuclear purely infinite $C^{*}$-algebras in the UCT class can be classified by their K-theoretic data. Katsura in [6] gave a sufficient condition on simple topological graph algebras such that they are purely infinite, and constructed all simple separable nuclear purely infinite $C^{*}$-algebras in the UCT class.

Graph algebras were firstly defined by Kumjian, Pask, Raeburn, and Renault in [12] using the groupoid $C^{*}$-algebras method. Topological graph algebras studied by Katsura (see [4), which are seen to be a generalization of graph algebras, were defined by using a modified version of Pimsner's construction (see [15]). A few people then tried to realize topological graph algebras as groupoid $C^{*}$-algebras, and these results can naturally be regarded as a generalization of Kumjian, Pask, Raeburn, and Renault's approach to graph algebras in [12. For example, Katsura in [7] showed that the topological graph algebra of a compact topological graph with a surjective range map is isomorphic to a RenaultDeaconu groupoid $C^{*}$-algebra. Yeend in [22] proved that every topological graph algebra is indeed an étale groupoid $C^{*}$-algebra. Kumjian and Li in [10] strengthened Yeend's result by showing that every topological graph algebra is indeed a Renault-Deaconu groupoid $C^{*}$-algebra (Yeend's result, and Kumjian and Li's result both cover a result of Brownlowe, Carlsen, and Whittaker from [3, Proposition 2.2]).

In this article, we give a sufficient condition on totally disconnected topological graphs such that their associated topological graph algebras are purely infinite. Our approach is different than Katsura's. Our strategy is that we deal with topological graph algebras under the groupoid model, and we apply Anantharaman-Delaroche's criterion in [1], which yields purely infinite étale groupoid $C^{*}$-algebras, to our settings.

Date: 24 Feb 2017.

2010 Mathematics Subject Classification. 46L05.

Key words and phrases. $C^{*}$-algebra; topological graph; Renault-Deaconu groupoid; groupoid $C^{*}$ algebra; pure infiniteness.

This research was supported by Research Center for Operator Algebras of East China Normal University and supported by Science and Technology Commission of Shanghai Municipality (STCSM), grant No. $13 \mathrm{dz} 2260400$. 
This paper is organized as follows. In Section 2, we give a background review on topological graph algebras and state the main theorem of [10]. In Section 3, we prove our main theorem, that is Theorem 3.11, which is a sufficient condition giving rise to purely infinite topological graph algebras. In Section 4, we give some remarks on our main theorem.

\section{PRELIMINARIES}

Throughout this paper, all the topological spaces are assumed to be second countable; and all the topological groupoids are assumed to be second countable. Our work in this article utilizes the Hilbert module, the $C^{*}$-correspondence, and the Cuntz-Pimsner algebra machinery. These materials can be referred to [8, 13, 15, 16, etc. This paper also involves groupoids, groupoid $C^{*}$-algebras, which can be found in [17].

2.1. Topological Graph Algebras. In this subsection, we recap some background about topological graphs and topological graph algebras from [4, 10].

Definition 2.1. Let $T$ be a locally compact Hausdorff space. Then $T$ is said to be totally disconnected if $T$ has an open base consisting of compact open subsets of $T$.

Definition 2.2 ([4, Definition 2.1]). A quadruple $E=\left(E^{0}, E^{1}, r, s\right)$ is called a topological graph if $E^{0}, E^{1}$ are locally compact Hausdorff spaces, $r: E^{1} \rightarrow E^{0}$ is a continuous map, and $s: E^{1} \rightarrow E^{0}$ is a local homeomorphism. In addition, $E$ is said to be totally disconnected if $E^{0}, E^{1}$ are both totally disconnected.

Definition $2.3([4])$. Let $E$ be a topological graph. For $x, y \in C_{c}\left(E^{1}\right), f \in C_{0}\left(E^{0}\right), e \in$ $E^{1}$, and for $v \in E^{0}$, define

$$
(x \cdot f)(e):=x(e) f(s(e)) ;(f \cdot x)(e):=f(r(e)) x(e) ; \text { and }\langle x, y\rangle_{C_{0}\left(E^{0}\right)}(v):=\sum_{s(e)=v} \overline{x(e)} y(e) .
$$

Then $C_{c}\left(E^{1}\right)$ is a right inner product $C_{0}\left(E^{0}\right)$-module with an adjointable left $C_{0}\left(E^{0}\right)$ action. Its completion $X(E)$ under the $\|\cdot\|_{C_{0}\left(E^{0}\right)}$-norm is called the graph correspondence associated to $E$. The Cuntz-Pimsner algebra of $X(E)$, which is denote by $\mathcal{O}(E)$, is called the topological graph algebra of $E$.

A subset $N$ of $E^{1}$ is called an $s$-section if $\left.s\right|_{N}: N \rightarrow s(N)$ is a homeomorphism with respect to the subspace topologies.

Define some useful subsets of $E^{0}$ as follows. Define

(1) $E_{\mathrm{sce}}^{0}:=E^{0} \backslash \overline{r\left(E^{1}\right)}$.

(2) $E_{\text {fin }}^{0}:=\left\{v \in E^{0}\right.$ : there exists an open neighborhood $N$ of $v$ such that $r^{-1}(\bar{N})$ is compact $\}$.

(3) $E_{\mathrm{rg}}^{0}:=E_{\mathrm{fin}}^{0} \backslash \overline{E_{\mathrm{sce}}^{0}}$.

(4) $E_{\mathrm{sg}}^{0}:=E^{0} \backslash E_{\mathrm{rg}}^{0}$.

For $n \geq 2$, define

$$
E^{n}:=\left\{\mu=\left(\mu_{1}, \ldots, \mu_{n}\right) \in \prod_{i=1}^{n} E^{1}: s\left(\mu_{i}\right)=r\left(\mu_{i+1}\right), i=1, \ldots, n-1\right\}
$$


regarded as a subspace of the product space $\prod_{i=1}^{n} E^{1}$. Define the finite-path space $E^{*}:=$ $\coprod_{n=0}^{\infty} E^{n}$ with the disjoint union topology. Define the infinite-path space

$$
E^{\infty}:=\left\{\mu \in \prod_{i=1}^{\infty} E^{1}: s\left(\mu_{i}\right)=r\left(\mu_{i+1}\right), i=1,2, \ldots\right\} .
$$

Denote the length of a path $\mu \in E^{*} \amalg E^{\infty}$ by $|\mu|$.

A finite path $\mu \in E^{*} \backslash E^{0}$ is called a cycle if $r(\mu)=s(\mu)$. The vertex $r(\mu)$ is called the base point of $\mu$. The cycle $\mu$ is said to be without entrances if $r^{-1}\left(r\left(\mu_{i}\right)\right)=\left\{\mu_{i}\right\}$, for $i=1, \ldots,|\mu|$. On the other hand, the cycle $\mu$ is said to have entrances if there exists $1 \leq i \leq|\mu|$ such that $r^{-1}\left(r\left(\mu_{i}\right)\right) \neq\left\{\mu_{i}\right\}$.

Definition 2.4 ([4, Definition 5.4]). Let $E$ be a topological graph. Then $E$ is said to be topologically free if the set of base points of cycles without entrances has empty interior.

Definition 2.5 ([10, Definitions $4.1,4.7]$ ). Let $E$ be a topological graph. Define the boundary path space to be $\partial E:=E^{\infty} \amalg\left\{\mu \in E^{*}: s(\mu) \in E_{\mathrm{sg}}^{0}\right\}$. For a subset $S \subset E^{*}$, define the cylinder set by $Z(S):=\{\mu \in \partial E$ : there exists $\alpha \in S$, such that $\mu=\alpha \beta\}$. Define a locally compact Hausdorff topology on $\partial E$ to be generated by the basic open sets $Z(U) \cap Z(K)^{c}$, where $U$ is an open set of $E^{*}$ and $K$ is a compact set of $E^{*}$.

By [10, Lemma 7.1], the one-sided shift map $\sigma: \partial E \backslash E_{\mathrm{sg}}^{0} \rightarrow \partial E$ is a local homeomorphism.

Definition 2.6. Let $E$ be a topological graph and let $v \in E^{0}$. Define the positive orbit space of $v$ (see [5, Definition 4.1]) by

$$
\operatorname{Orb}^{+}(v):=\left\{w \in E^{0}: \text { there exists } \nu \in E^{*} \text {, such that } r(\nu)=w, s(\nu)=v\right\} .
$$

$E$ is said to be cofinal if for any $\mu \in \partial E, \operatorname{Orb}^{+}(r(\mu)) \cup\left(\bigcup_{i=1}^{|\mu|} \operatorname{Orb}^{+}\left(s\left(\mu_{i}\right)\right)\right)$ is dense in $E^{0}$.

Remark 2.7. The cofinality for topological graphs is a generalization of the cofinality for directed graphs (see [11, 12]). Let $E$ be a directed graph, the cofinality of $E$ means that for any $v \in E^{0}$, and for any $\mu \in \partial E$, there exists $\nu \in E^{*}$, such that $r(\nu)=v, s(\nu) \in$ $\{r(\mu)\} \bigcup\left(\bigcup_{i=1}^{|\mu|}\left\{s\left(\mu_{i}\right)\right\}\right)$.

Theorem 2.8 ([5, Proposition 8.9, Theorem 8.12]). Let E be a topological graph. Then $\mathcal{O}(E)$ is simple if and only if $E$ is topologically free and cofinal.

2.2. Groupoid $C^{*}$-algebras. In this subsection, we state one of the main theorems of [10].

A topological groupoid is called a locally compact groupoid if its topology is locally compact Hausdorff. A locally compact groupoid is said to be étale if its range map is a local homeomorphism.

Let $\Gamma$ be a locally compact groupoid and let $N \subset \Gamma$. Then $N$ is called an s-section if $\left.s\right|_{N}: N \rightarrow s(N)$ is a homeomorphism with respect to the subspace topologies; $N$ is called an $r$-section if $\left.r\right|_{N}: N \rightarrow r(N)$ is a homeomorphism with respect to the subspace topologies; and $N$ is called a bisection if $\left.s\right|_{N},\left.r\right|_{N}$ are both homeomorphisms with respect to the subspace topologies. 
Definition 2.9 ([18, Definition 2.4]). Let $T$ be a locally compact Hausdorff space and let $\sigma: \operatorname{dom}(\sigma) \rightarrow \operatorname{ran}(\sigma)$ be a partial local homeomorphism. Define the Renault-Deaconu groupoid $\Gamma(T, \sigma)$ as follows:

$$
\begin{aligned}
\Gamma(T, \sigma):=\left\{\left(t_{1}, k_{1}-k_{2}, t_{2}\right) \in T \times \mathbb{Z} \times T:\right. & k_{1}, k_{2} \geq 0, t_{1} \in \operatorname{dom}\left(\sigma^{k_{1}}\right), \\
& \left.t_{2} \in \operatorname{dom}\left(\sigma^{k_{2}}\right), \sigma^{k_{1}}\left(t_{1}\right)=\sigma^{k_{2}}\left(t_{2}\right)\right\} .
\end{aligned}
$$

Define the unit space $\Gamma^{0}:=\{(t, 0, t): t \in T\}$. For $\left(t_{1}, n, t_{2}\right),\left(t_{2}, m, t_{3}\right) \in \Gamma(T, \sigma)$, define the multiplication, the inverse, the source and the range map by

$$
\begin{aligned}
\left(t_{1}, n, t_{2}\right)\left(t_{2}, m, t_{3}\right) & :=\left(t_{1}, n+m, t_{3}\right) ;\left(t_{1}, n, t_{2}\right)^{-1}:=\left(t_{2},-n, t_{1}\right) \\
r\left(t_{1}, n, t_{2}\right) & :=\left(t_{1}, 0, t_{1}\right) ; s\left(t_{1}, n, t_{2}\right):=\left(t_{2}, 0, t_{2}\right) .
\end{aligned}
$$

Define the topology on $\Gamma(T, \sigma)$ to be generated by the basic open set

$$
\mathcal{U}\left(U, V, k_{1}, k_{2}\right):=\left\{\left(t_{1}, k_{1}-k_{2}, t_{2}\right): t_{1} \in U, t_{2} \in V, \sigma^{k_{1}}\left(t_{1}\right)=\sigma^{k_{2}}\left(t_{2}\right)\right\},
$$

where $U \subset \operatorname{dom}\left(\sigma^{k_{1}}\right), V \subset \operatorname{dom}\left(\sigma^{k_{2}}\right)$ are open in $T, \sigma^{k_{1}}$ is injective on $U$, and $\sigma^{k_{2}}$ is injective on $V$.

Definition 2.10 ([10, Definition 7.5]). Let $E$ be a topological graph. Define the boundary path groupoid to be the Renault-Deaconu groupoid

$$
\Gamma(\partial E, \sigma):=\left\{(\mu, k-l, \nu) \in \partial E \times \mathbb{Z} \times \partial E: \mu \in \operatorname{dom}\left(\sigma^{k}\right), \nu \in \operatorname{dom}\left(\sigma^{l}\right), \sigma^{k}(\mu)=\sigma^{l}(\nu)\right\} .
$$

The following theorem is a special case of [10, Theorem 7.6].

Theorem 2.11. Let $E$ be a topological graph. Then $\mathcal{O}(E)$ is isomorphic to the groupoid $C^{*}$-algebra $C^{*}(\Gamma(\partial E, \sigma))$.

\section{Sufficient Conditions of Purely Infinite Topological Graph Algebras}

Definition $3.1([20])$. Let $A$ be a $C^{*}$-algebra. Then $A$ is said to be purely infinite if every nonzero hereditary $C^{*}$-subalgebra of $A$ contains an infinite projection.

We firstly prove the following two technical lemmas.

Lemma 3.2. Let $E$ be a topological graph. Fix an open set $U \subset E^{*}$, and fix a compact set $K \subset E^{*}$ satisfying $Z(U) \cap Z(K)^{c} \neq \emptyset$. Write $K=\bigcup_{i=0}^{k}\left(K \cap E^{i}\right)$ for some $k \geq 0$. Then for any $\mu \in Z(U) \cap Z(K)^{c}$ with $|\mu| \geq k$, there exists an open subset $V$ of $E^{*}$ such that $\mu \in Z(V) \subset Z(U) \cap Z(K)^{c}$. In particular, if $|\mu|=k$, then $V$ can be chosen to be an open neighborhood of $\mu$ in $E^{|\mu|}$.

Proof. We prove the first statement. Write $\mu=\alpha \beta$ where $\alpha \in U$.

Case $1 . K=\emptyset$. Let $V:=U$. Then we are done.

Case 2. $K \neq \emptyset$ and $K \subset E^{0}$. Then $r(\mu) \notin K$. Take an open neighborhood $N$ of $r(\mu)$ which does not intersect with $K$. Let $V:=\left(r^{|\alpha|}\right)^{-1}(N) \cap U$. We have $\mu \in Z(V) \subset$ $Z(U) \cap Z(K)^{c}$.

Case 3. $K \neq \emptyset$ and $K \not \subset E^{0}$. Then $k \geq 1$. Since $\mu \in Z(K)^{c}$, then for $1 \leq i \leq k$ there exists an open neighborhood $N_{i} \subset E^{i}$ of $\mu_{1} \ldots \mu_{i}$ such that $N_{i}$ does not intersect with 
$K \cap E^{i}$ and $r\left(N_{i}\right)$ does not intersect with $K \cap E^{0}$. Let

$$
V:= \begin{cases}\left(\bigcap_{i=1}^{k-1}\left(N_{i} \times E^{k-i}\right)\right) \cap N_{k} \cap\left(\left(U \cap E^{|\alpha|}\right) \times E^{k-|\alpha|}\right) & \text { if } k>|\alpha|>0 \\ \left.\bigcap_{i=1}^{k}\left(N_{i} \times E^{|\alpha|-i}\right)\right) \cap\left(U \cap E^{|\alpha|}\right) & \text { if }|\alpha|>k \\ \left.\bigcap_{i=1}^{k-1}\left(N_{i} \times E^{k-i}\right)\right) \cap N_{k} \cap\left(U \cap E^{|\alpha|}\right) & \text { if }|\alpha|=k \\ \left.\bigcap_{i=1}^{k-1}\left(N_{i} \times E^{k-i}\right)\right) \cap N_{k} \cap\left(r^{k}\right)^{-1}\left(U \cap E^{|\alpha|}\right) & \text { if }|\alpha|=0 .\end{cases}
$$

Then $V$ is an open subset of $E^{\max \{|\alpha|, k\}}$ and $\mu \in Z(V) \subset Z(U) \cap Z(K)^{c}$.

We prove the second statement. If $K=\emptyset$, let

$$
V:= \begin{cases}\left(U \cap E^{|\alpha|}\right) \times E^{|\beta|} & \text { if }|\alpha|>0,|\beta|>0 \\ \left(r^{k}\right)^{-1}(U) & \text { if }|\alpha|=0,|\beta|>0 \\ U \cap E^{k} & \text { if }|\beta|=0 .\end{cases}
$$

Then $V$ is an open neighborhood of $\mu$ in $E^{|\mu|}$, and $Z(V) \subset Z(U) \cap Z(K)^{c}$. If $K \neq \emptyset$, then it follows directly from the above construction.

Lemma 3.3. Let $E$ be a topological graph. Suppose that $E_{\mathrm{sce}}^{0}=\emptyset$. Then for any open set $U \subset E^{*}$ and any compact set $K \subset E^{*}$ satisfying $Z(U) \cap Z(K)^{c} \neq \emptyset$, there exists an open subset $V$ of $E^{*}$ such that $\emptyset \neq Z(V) \subset Z(U) \cap Z(K)^{c}$.

Proof. The assumption $E_{\mathrm{sce}}^{0}=\emptyset$ gives $E_{\mathrm{sg}}^{0}=E^{0} \backslash E_{\mathrm{fin}}^{0} E^{0}=E_{\mathrm{rg}}^{0} \cup\left(E_{\mathrm{fin}}^{0}\right)^{c}$. Fix an open set $U \subset E^{*}$ and fix a compact set $K \subset E^{*}$ satisfying $Z(U) \cap Z(K)^{c} \neq \emptyset$. Write $K=$ $\bigcup_{i=0}^{k}\left(K \cap E^{i}\right)$, for some $k \geq 0$. Fix $\mu \in Z(U) \cap Z(K)^{c}$. If $|\mu| \geq k$ then by Lemma 3.2 we are done. So we may assume that $|\mu|<k$. By Lemma 3.2, there exists an open neighborhood $V \subset E^{|\mu|}$ of $\mu$ such that $Z(V) \subset Z(U) \cap Z\left(\bigcup_{i=0}^{|\mu|}\left(K \cap E^{i}\right)\right)^{c}$. Then $s(V)$ is an open neighborhood of $s(\mu)$. Since $\mu \in \partial E$ and $E_{\mathrm{sg}}^{0}=E^{0} \backslash E_{\text {fin }}^{0}$, we have $s(\mu) \in E^{0} \backslash E_{\text {fin }}^{0}$. Consider the set

$$
F:=\left\{\alpha_{|\mu|+1} \in E^{1}: \alpha \in K,|\alpha| \geq|\mu|+1\right\}
$$

which is a compact subset of $E^{1}$. Then there exists $e \in r^{-1}(s(V))$ such that $e \notin F$. Take an open neighborhood $W \subset E^{1}$ of $e$ which does not intersect with $F$. Let

$$
O:= \begin{cases}(V \times W) \cap E^{|\mu|+1} & \text { if }|\mu|>0 \\ W & \text { if }|\mu|=0 .\end{cases}
$$

So $\emptyset \neq Z(O) \subset Z(U) \cap Z(K)^{c}$.

Definition 3.4 ([1, Page 202]). Let $\Gamma$ be an étale groupoid. Then $\Gamma$ is said to be essentially free if the set of elements in $\Gamma^{0}$ whose isotropy group are trivial form a dense subset of $\Gamma^{0}$.

Lemma 3.5. Let $T$ be a locally compact Hausdorff space and let $\sigma: \operatorname{dom}(\sigma) \rightarrow \operatorname{ran}(\sigma)$ be a partial local homeomorphism. Then the Renault-Deaconu groupoid $\Gamma(T, \sigma)$ is essentially free if and only if the set $\{t \in T: t, \sigma(t), \ldots$ are distinct $\}$ is dense in $T$.

Proof. It is straightforward to see.

Definition 3.6. Let $E$ be a topological graph. Then $E$ is said to be essentially free if the boundary path groupoid $\Gamma(\partial E, \sigma)$ is essentially free. 
The following proposition is a generalization of [11, Lemma 3.4].

Proposition 3.7. Let $E$ be a topological graph. Then $E$ is topologically free if and only if $E$ is essentially free.

Proof. First of all, suppose that $E$ is not essentially free. We aim to show that $E$ is not topologically free. Since $E$ is not essentially free, there exists a nonempty open set $N \subset \partial E$ which does not intersect with $\{\mu \in \partial E: \mu, \sigma(\mu), \ldots$ are distinct $\}$ due to Lemma 3.5. For each $0 \leq p<q$, define a closed subset of $\partial E$ to be $B_{p, q}:=\{\mu \in$ $\left.\partial E: \sigma^{p}(\mu)=\sigma^{q}(\nu)\right\}$. For each $0 \leq p<q$, let $A_{p, q}:=B_{p, q} \cap N$, then $A_{p, q}$ is a closed subset of the subspace $N$. Notice that $N=\bigcup_{0 \leq p<q} A_{p, q}$ because $N$ does not intersect with $\{\mu \in \partial E: \mu, \sigma(\mu), \ldots$ are distinct $\}$. By the Baire's category theorem, there exists a nonempty open subset $O$ of $N\left(O\right.$ is also open in $\partial E$ ) contained in $A_{p_{0}, q_{0}}$ for some $1 \leq p_{0}<q_{0}$. By Definition [2.5, there exist an open set $U \subset E^{*}$ and a compact set $K \subset E^{*}$ such that $\emptyset \neq Z(U) \cap Z(K)^{c} \subset O$. By Lemma 3.2, there exist $n \geq 0$ and an open subset $V \subset E^{n}$ such that $\emptyset \neq Z(V) \subset O$. We may assume that $n \neq 0$. Let $W:=\left(V \times\left(r^{p}\right)^{-1}(s(V))\right) \cap E^{n+p_{0}}$. Then $Z(W) \subset Z(V)$. So $Z(W) \subset A_{p_{0}, q_{0}}$. We deduce that every $\mu \in Z(W)$ satisfies that $\sigma^{n+p_{0}}(\mu)=\sigma^{n+q_{0}}(\mu)$ since $\mu \in A_{p_{0}, q_{0}}$. [4, Proposition 2.8] assures that for each $\alpha \in W$ there exists $\mu \in Z(W)$ such that $\mu=\alpha \beta$. We then conclude that $s(W)$ is an open subset of $E^{0}$ consisting of base points of cycles. We claim that $s(W)$ consists of base points of cycles without entrances. Suppose not, for a contradiction. We obtain two infinite paths $\alpha \beta, \alpha \beta^{\prime} \in Z(W)$, where $\alpha \in W, \beta_{1} \neq \beta_{2}$. Since $\nu:=\beta_{1} \ldots \beta_{q_{0}-p_{0}}, \nu^{\prime}:=\beta_{1}^{\prime} \ldots \beta_{q_{0}-p_{0}}^{\prime}$ are cycles, $\alpha \nu \nu^{\prime} \nu \nu^{\prime} \cdots \in Z(W) \notin A_{p_{0}, q_{0}}$, which is a contradiction. Hence $s(W)$ is an open subset of $E^{0}$ consisting of base points of cycles. Therefore $E$ is not topologically free.

Conversely suppose that $E$ is essentially free. Suppose that $E$ is not topologically free, for a contradiction. By Lemma 3.5, the set $\{\mu \in \partial E: \mu, \sigma(\mu), \ldots$ are distinct $\}$ is dense in $\partial E$. By [5, Proposition 6.12], there exist a nonempty open set $V \subset E^{0}$ consisting of base points of cycles without entrances, and a homeomorphism $\sigma$ on $V$ such that $\sigma=r \circ\left(\left.s\right|_{r^{-1}(V)}\right)^{-1}$. By [4, Proposition 2.8], $V \subset E_{\mathrm{rg}}^{0}$. Fix a vertex $v \in V$. Let $\nu$ be the unique simple cycle such that $r(\nu)=v$. By the assumption, there is a convergent sequence $\left(\nu^{(n)}\right)_{n=1}^{\infty} \subset\{\mu \in \partial E: \mu, \sigma(\mu), \ldots$ are distinct $\}$ with the limit $\nu$. By [10, Lemma 4.8], $r\left(\nu^{(n)}\right) \rightarrow r(\nu)=v$, and so there exists $N \geq 1$ such that $r\left(\nu^{(N)}\right) \in V$. Let $\alpha$ be the unique simple cycle with $r(\alpha)=r\left(\nu^{(N)}\right)$. Since $V$ consists of base points of cycles without entrances and $V \subset E_{\mathrm{rg}}^{0}$, we deduce that $\nu^{(N)}=\alpha \alpha \cdots$, which is a contradiction. So $E$ is topologically free.

Definition 3.8 ([1, Definition 2.1]). Let $\Gamma$ be an étale groupoid. Then $\Gamma$ is said to be locally contracting if for any nonempty open set $U \subset \Gamma^{0}$, there exist an open subset $V \subset U$ and an open bisection $N \subset \Gamma$ such that $\bar{V} \subset s(N)$ and $\left.s \circ r\right|_{N^{-1}} ^{-1}(\bar{V}) \varsubsetneqq V$.

Definition 3.9. Let $E$ be a topological graph. Then $E$ is said to be locally contracting if the boundary path groupoid $\Gamma(\partial E, \sigma)$ is locally contracting.

Definition 3.10. Let $E$ be a topological graph and let $v \in E^{0}$. Then $v$ is said to connect to a cycle if there exists $u \in E^{0}$ such that $v \in \mathrm{Orb}^{+}(u)$ and $u$ is the base point of a cycle.

The following theorem is a generalization of partial results from [11, Theorem 3.9]. 
Theorem 3.11. Let $E$ be a topologically free totally disconnected topological graph. Suppose that the following subset of $E^{0}$ is dense in $E^{0}$.

$B:=\{v: v$ connects to a cycle $\mu$ with entrances such that any open neighborhood $N$ of $\mu$ contains an open neighborhood $U$ of $\mu$ with $\left.r^{|\mu|}(U) \subset s^{|\mu|}(U)\right\}$.

Then $E$ is essentially free and locally contracting. Hence $\mathcal{O}(E)$ is purely infinite.

Proof. Since every cycle of $E$ has entrances, $E$ is topologically free. So $E$ is essentially free by Proposition 3.7 .

We claim that $E_{\mathrm{sce}}^{0}=\emptyset$. Suppose that $E_{\mathrm{sce}}^{0} \neq \emptyset$, for a contradiction. Fix $v \in E_{\mathrm{sce}}^{0}$. Then there exists an open neighborhood $V$ of $v$ not intersecting with $\overline{r\left(E^{1}\right)}$. Since $B$ is dense in $E^{0}, V \cap B \neq \emptyset$ which is a contradiction. So $E_{\text {sce }}^{0}=\emptyset$ and we finish proving the claim.

Now we prove that $E$ is locally contracting. Fix a nonempty open set $N \subset \partial E$. By Lemma 3.3 there exists a nonempty open subset $U$ of $E^{*}$ such that $\emptyset \neq Z(U) \subset N$. Then $s(U)$ is a nonempty open subset of $E^{0}$. Since $B$ is dense in $E^{0}$, there exist $\mu \nu e \beta, \mu \nu \alpha e \beta \in$ $\partial E$ such that $\mu \in U, \alpha$ is a cycle, and $e \neq \alpha_{1}$. We may assume that $|\mu \nu|>0$ (the case $|\mu \nu|=0$ would follow a similar argument). Take a compact open $s^{|\mu \nu|}$-sections $O_{1}$ such that $\mu \nu \in O_{1}$ and $Z\left(O_{1}\right) \subset Z(U)$; and take compact open $s^{|\alpha|}$-sections $O_{2}$ such that $\alpha \in$ $O_{2}, r^{|\alpha|}\left(O_{2}\right) \subset s^{|\mu \nu|}\left(O_{1}\right) \cap s^{|\alpha|}\left(O_{2}\right), e \notin\left\{\alpha_{1}^{\prime}: \alpha^{\prime} \in O_{2}\right\}$. Define a compact open bisection of the boundary path groupoid $\Gamma(\partial E, \sigma)$ by $S:=\mathcal{U}\left(Z\left(\left(O_{1} \times O_{2}\right) \cap E^{|\mu \nu \alpha|}\right), Z\left(O_{1}\right),|\mu \nu \alpha|,|\mu \nu|\right)$. Define a nonempty compact open subset of $N$ by $W:=s(S)$. Then $\bar{W} \subset s(S)$. For any $\mu^{\prime} \nu^{\prime}, \mu^{\prime \prime} \nu^{\prime \prime} \in O_{1}, \alpha^{\prime} \in O_{2}, \beta \in \partial E$ such that $\left(\mu^{\prime} \nu^{\prime} \alpha^{\prime} \beta,|\mu \nu \alpha|-|\mu \nu|, \mu^{\prime \prime} \nu^{\prime \prime} \beta\right) \in S$, there exist unique $\mu^{\prime \prime \prime}, \nu^{\prime \prime \prime} \in O_{1}, \alpha^{\prime \prime \prime} \in O_{2}$ such that $\mu^{\prime \prime \prime} \nu^{\prime \prime \prime} \alpha^{\prime \prime \prime} \alpha^{\prime} \beta \in Z\left(Z\left(\left(O_{1} \times O_{2}\right) \cap E^{|\mu \nu \alpha|}\right)\right)$. So $\mu^{\prime} \nu^{\prime} \alpha^{\prime} \beta=s\left(\mu^{\prime \prime \prime} \nu^{\prime \prime \prime} \alpha^{\prime \prime \prime} \alpha^{\prime} \beta,|\mu \nu \alpha|-|\mu \nu|, \mu^{\prime} \nu^{\prime} \alpha^{\prime} \beta\right) \in W$. Hence $s \circ r_{S^{-1}}^{-1}(\bar{W}) \subset W$. Pick up an arbitrary $\gamma \in s(e) \partial E$ (see [4, Proposition 2.8]). Then $\mu \nu e \gamma \in W$ but $\mu \nu e \gamma \notin s \circ r_{S^{-1}}^{-1}(\bar{W})$ because $e \notin\left\{\alpha_{1}^{\prime}: \alpha^{\prime} \in O_{2}\right\}$. Therefore $s \circ r_{S^{-1}}^{-1}(\bar{W}) \varsubsetneqq W$. By Definition [3.9, $\Gamma(\partial E, \sigma)$ is locally contracting.

By Theorem 2.11, $\mathcal{O}(E)$ is isomorphic with $C^{*}(\Gamma(\partial E, \sigma))$. Since $E$ is essentially free and locally contracting, [1, Proposition 2.4] gives $\mathcal{O}(E)$ is purely infinite.

Corollary 3.12. Let $E$ be a totally disconnected topological graph such that $\mathcal{O}(E)$ is simple. Suppose that there exists a cycle $\mu$ with entrances such that any open neighborhood $N$ of $\mu$ contains an open neighborhood $U$ of $\mu$ with $r^{|\mu|}(U) \subset s^{|\mu|}(U)$. Then $E$ is essentially free and locally contracting. Hence $\mathcal{O}(E)$ is purely infinite.

Proof. Since $\mathcal{O}(E)$ is simple, by Theorem $2.8 E$ is topologically free. By Proposition 3.7 $E$ is essentially free. Fix $v \in E^{0}$ and fix an open neighborhood $V$ of $v$. Since $\mathcal{O}(E)$ is simple, by Theorem $2.8 E$ is cofinal. So $\bigcup_{i=1}^{|\mu|} \operatorname{Orb}^{+}\left(s\left(\mu_{i}\right)\right)$ is dense in $E^{0}$. Hence $V \cap\left(\bigcup_{i=1}^{N} \operatorname{Orb}^{+}\left(s\left(\mu_{i}\right)\right)\right) \neq \emptyset$. Thus $V \cap B \neq \emptyset$ (see Theorem 3.11). Theorem 3.11 implies that $E$ is locally contracting. Therefore [1, Proposition 2.4] yields that $\mathcal{O}(E)$ is purely infinite.

\section{Concluding Remarks}

Katsura in [6] defined a concept called contracting topological graphs which can provide purely infinite topological graph algebras. We recall the definition of contracting topological graphs and state Katsura's result. 
Definition 4.1 ([6, Definition 2.3]). Let $E$ be a topological graph. A nonempty precompact open set $V \subset E^{0}$ is said to be contracting if there exists a finite family of nonempty open sets $\left\{U_{i} \subset E^{n_{i}}: n_{i} \geq 1\right\}_{i=1}^{k}$ satisfying the following.

(1) $r\left(U_{i}\right) \subset V, i=1, \ldots, k$;

(2) for $i \neq j, n_{i} \leq n_{j}$, we have $\left\{\left(\mu_{1}, \ldots, \mu_{n_{i}}\right):\left(\mu_{1}, \ldots, \mu_{n_{i}}\right) \in U_{i}, \mu \in E^{n_{j}}\right\}=\emptyset$; and

(3) $\bar{V} \varsubsetneqq \bigcup_{i=1}^{k} s\left(U_{i}\right)$.

Moreover, $E$ is said to be contracting at a vertex $v \in E^{0}$ if $\overline{\operatorname{Orb}^{+}(v)}=E^{0}$ and every open neighborhood of $v$ contains a contracting open set. Furthermore, $E$ is said to be contracting if $E$ is contracting at some vertex in $E^{0}$.

Theorem 4.2 ([6, Theorem A]). Let $E$ be a topological graph. Suppose that $\mathcal{O}(E)$ is simple. If $E$ is contracting then $\mathcal{O}(E)$ is purely infinite.

We provide two examples which indicate that both of pure infiniteness conditions for simple totally disconnected topological graph algebras in Corollary 3.12 and Theorem 4.2 are not comparable.

Example 4.3. Define $E^{0}:=\{v\} ; E^{1}:=\left\{e_{0}, e_{1}\right\}$. Then $\mathcal{O}(E) \cong \mathcal{O}_{2}$ which is simple and purely infinite. Notice that $e_{0}$ is a cycle with entrances and $\left\{e_{0}\right\}$ is an open neighborhood of $e_{0}$ with $r\left(\left\{e_{0}\right\}\right) \subset s\left(\left\{e_{0}\right\}\right)$. So the assumption of Corollary 3.12 is satisfied. However, it is easy to see that this graph is not contracting. Therefore the assumption of Theorem 4.2 is not satisfied. Furthermore, Katsura in [6, Remark 2.8] asked that whether the converse of Theorem 4.2 is true and this is a counterexample of the question.

Example 4.4. Define $E^{0}:=\{v\} ; E^{1}:=\left\{e_{0}, e_{1}\right\}$. This time we consider the dual graph of $\widehat{E}:=\left(\prod_{n=1}^{\infty}\{0,1\}, \prod_{n=1}^{\infty}\{0,1\}, \mathrm{id}, \sigma\right)$ where $\sigma$ is the one-sided shift. By [10, Theorem 7.5], $\mathcal{O}(\widehat{E}) \cong \mathcal{O}(E)$ so $\mathcal{O}(\widehat{E})$ is simple and purely infinite. Since every cycle of $\widehat{E}$ has no entrances, the assumption of Corollary 3.12 is not satisfied. On the other hand, $\overline{\mathrm{Orb}^{+}(000 \ldots)}=\widehat{E}^{0}$ and $Z(\underbrace{0 \cdots 0}_{n}) \subset \widehat{E}^{0}$ is contracting for all $n \geq 1$. To see that $Z(\underbrace{0 \cdots 0}_{n})$ is contracting, let $U_{1}:=Z(\underbrace{0 \cdots 0}_{n+1}) \subset \widehat{E}^{1}, U_{2}:=Z(\underbrace{0 \cdots 0}_{n} 1) \subset \widehat{E}^{1}$. It is straightforward to check that $\left\{U_{1}, U_{2}\right\}$ satisfies Conditions (11)-(13) of Definition 4.1, So $Z(\underbrace{0 \cdots 0}_{n})$ is contracting. Hence $E$ is contracting and the assumption of Theorem 4.2 is satisfied.

Yeend in [22] showed that topological 1-graph $C^{*}$-algebras coincide with topological graph algebras (see also [10]). Later, Renault, Sims, Williams, and Yeend in [19] provided a sufficient condition for simple compactly-aligned topological higher-rank graph $C^{*}$-algebras to be purely infinite. In the following we interpret their condition in the topological graph setting and present their result.

Definition 4.5 ([19, Definition 5.7]). Let $E$ be a topological graph. A nonempty precompact open set $U \subset E^{0}$ is said to be contracting if there exist $0 \leq n<m$, a nonempty precompact open $s^{n}$-section $Y_{n}$, and a nonempty precompact open $s^{m}$-section $Y_{m}$, such that

(1) $s^{m}\left(Y_{m}\right)=s^{n}\left(Y_{n}\right)$;

(2) $\overline{r\left(Y_{m}\right)} \subset r\left(Y_{n}\right)=U$;

(3) for $\mu \in Y_{m}, \nu \in Y_{n}$ with $r^{m}(\mu)=r^{n}(\nu)$, there exists $\gamma \in E^{m-n}$ such that $\mu=\nu \gamma$; 
(4) there exists a nonempty open subset $W$ of $Y_{n} E^{*}$ such that $\left\{\nu_{1} \ldots \nu_{n}: \nu \in W\right\}=Y_{n}$ and that for $\mu \in Y_{m}, \nu \in W$ there is no $\gamma \in E^{*}$ satisfying $\mu=\nu \gamma$ or $\nu=\mu \gamma$.

Theorem 4.6 ([19, Proposition 5.8]). Let $E$ be a topological graph. Suppose that $\mathcal{O}(E)$ is simple and that for any $v \in E^{0}$, there exist $n \geq 0$ and an open subset $U$ of $E^{n}$ satisfying that $v \in r(U)$ and $s(U)$ is contracting in the sense of Definition 4.5. Then $\mathcal{O}(E)$ is purely infinite.

The disadvantage of this theorem is that in order to prove the pure infiniteness of a topological graph algebra, one has to check the contracting condition for every vertex.

Overall, both criteria of Katsura and Renault-Sims-Williams-Yeend for topological graph algebras being purely infinite relies on the assumption of the given topological graph algebras being simple. Our approach does not but pay the price that we have to assume that the starting topological graphs are totally disconnected.

Finally, we consider a compact totally disconnected topological graph $E$ such that the range, source map are surjective and $\mathcal{O}(E)$ is simple. Schafhauser recently proved that $\mathcal{O}(E)$ is finite if and only if the source map is injective (see [21, Theorem 6.7]). On the other hand, it is hard to get a completely graphic characterization for $\mathcal{O}(E)$ to be purely infinite. However, by combining the recent work of Brown, Clark, and Sierakowski in [2] and the work of Kumjian and Li [10], we are able to reduce the problem by only considering the projections on the vertex space. More precisely, $\mathcal{O}(E)$ is purely infinite if and only if every nonzero projection on $C\left(E^{0}\right)$ is infinite.

\section{REFERENCES}

[1] C. Anantharaman-Delaroche, Purely infinite $C^{*}$-algebras arising from dynamical systems, Bull. Soc. Math. France 125 (1997), 199-225.

[2] J. Brown, L.O. Clark, and A. Sierakowski, Purely infinite $C^{*}$-algebras associated to étale groupoids, Ergodic Theory Dynam. Systems 35 (2015), 2397-2411.

[3] N. Brownlowe, T.M. Carlsen, and M.F. Whittaker, Graph algebras and orbit equivalence, preprint, arXiv:1410.2308.

[4] T. Katsura, A class of $C^{*}$-algebras generalizing both graph algebras and homeomorphism $C^{*}$-algebras I. Fundamental results, Trans. Amer. Math. Soc. 356 (2004), 4287-4322.

[5] T. Katsura, A class of $C^{*}$-algebras generalizing both graph algebras and homeomorphism $C^{*}$-algebras III. Ideal structures, Ergodic Theory Dynam. Systems 26 (2006), 1805-1854.

[6] T. Katsura, A class of $C^{*}$-algebras generalizing both graph algebras and homeomorphism $C^{*}$-algebras. IV. Pure infiniteness, J. Funct. Anal. 254 (2008), 1161-1187.

[7] T. Katsura, Cuntz-Krieger algebras and $C^{*}$-algebras of topological graphs, Acta Appl. Math. 108 (2009), 617-624.

[8] T. Katsura, On $C^{*}$-algebras associated with $C^{*}$-correspondences, J. Funct. Anal. 217 (2004), 366401.

[9] E. Kirchberg, The classification of purely infinite $C^{*}$-algebras using Kasparov's theory, preprint, 1994.

[10] A. Kumjian and H. Li, Twisted topological graph algebras are twisted groupoid $C^{*}$-algebras, J. Operator Theory, to appear, arXiv:1507.04449.

[11] A. Kumjian, D. Pask, and I. Raeburn, Cuntz-Krieger algebras of directed graphs, Pacific J. Math. 184 (1998), 161-174.

[12] A. Kumjian, D. Pask, I. Raeburn, and J. Renault, Graphs, groupoids, and Cuntz-Krieger algebras, J. Funct. Anal. 144 (1997), 505-541.

[13] E.C. Lance, Hilbert $C^{*}$-modules, A toolkit for operator algebraists, Cambridge University Press, Cambridge, 1995, x+130.

[14] N.C. Phillips, A classification theorem for nuclear purely infinite simple $C^{*}$-algebras, Doc. Math. 5 (2000), 49-114. 
[15] M.V. Pimsner, A class of $C^{*}$-algebras generalizing both Cuntz-Krieger algebras and crossed products by Z, Fields Inst. Commun., 12, Free probability theory (Waterloo, ON, 1995), 189-212, Amer. Math. Soc., Providence, RI, 1997.

[16] I. Raeburn and D.P. Williams, Morita equivalence and continuous-trace $C^{*}$-algebras, American Mathematical Society, Providence, RI, 1998, xiv+327.

[17] J. Renault, A groupoid approach to $C^{*}$-algebras, Springer, Berlin, 1980, ii +160 .

[18] J. Renault, Cuntz-like algebras, Operator theoretical methods (Timişoara, 1998), 371-386, Theta Found., Bucharest, 2000.

[19] J. Renault, A. Sims, D.P. Williams, and T.Yeend, Uniqueness theorems for topological higher-rank graph $C^{*}$-algebras, preprint, arXiv:0906.0829.

[20] M. Rørdam and E. Størmer, Classification of nuclear $C^{*}$-algebras Entropy in operator algebras, Operator Algebras and Non-commutative Geometry, 7, Springer-Verlag, Berlin, 2002, x+198.

[21] C.P. Schafhauser, Finiteness properties of certain topological graph algebras, Bull. Lond. Math. Soc. 47 (2015), 443-454.

[22] T. Yeend,Topological higher-rank graphs and the $C^{*}$-algebras of topological 1-graphs, Contemp. Math., 414, Operator theory, operator algebras, and applications, 231-244, Amer. Math. Soc., Providence, RI, 2006.

E-mail address: lihui8605@hotmail.com

Research Center for Operator Algebras and Shanghai Key Laboratory of Pure MathEmatics and Mathematical Practice, Department of Mathematics, East China Normal University, 3663 Zhongshan North Road, Putuo District, Shanghai 200062, China 